\title{
Power dissipation in HTS coated conductor coils under the simultaneous action of $A C$ and DC currents and fields
}

\author{
Boyang Shen ${ }^{1}$, Chao $\mathrm{Li}^{1}$, Jianzhao Geng ${ }^{1}$, Xiuchang Zhang ${ }^{1}$, James Gawith ${ }^{1}$, Jun Ma ${ }^{1}$, Yingzhen Liu ${ }^{2}$, \\ Francesco Grilli ${ }^{2}$, and T. A. Coombs ${ }^{1}$
}

\begin{abstract}
${ }^{1}$ Electrical Engineering Division, Department of Engineering, University of Cambridge, Cambridge CB3 0FA, U.K.
${ }^{2}$ Institute for Technical Physics, Karlsruhe Institute of Technology (KIT), 76131 Karlsruhe, Germany.

E-mail: bs506@cam.ac.uk
\end{abstract}

\begin{abstract}
This paper presents a comprehensive AC loss study of a circular HTS coil. The AC losses from a circular double pancake coil were measured using the electrical method. A 2D axisymmetric $\mathrm{H}$-formulation model using FEM package COMSOL Multiphysics has been established, which was able to make consistency with the real circular coil used in the experiment. To model a circular HTS coil, a 2D axisymmetric model provided better accuracy than a general 2D model, and was also more efficient than a 3D model. Three scenarios have been analysed: Scenario 1 AC transport current and DC magnetic field (experiment and simulation); Scenario 2 DC transport current and AC magnetic field (simulation); Scenario 3 AC transport current and AC magnetic field (simulation and experimental data support). The angular dependence analysis on the coil under the magnetic field with the different orientation angle $\theta$ has been carried out for all three scenarios. For Scenario 3, we investigated the effect of relative phase difference $\Delta \varphi$ between $\mathrm{AC}$ current and AC field on the total AC loss of the coil. To summarise, we have carried out a current/field/angle/phase dependent AC loss $(\boldsymbol{I}, \boldsymbol{B}, \theta, \Delta \varphi)$ study of circular HTS coil, which could potentially benefit the future design and research of HTS AC systems.
\end{abstract}

Keywords: High Temperature Superconductor (HTS), HTS coil, AC loss, Angular dependence, Phase difference.

\section{Introduction}

Coils and cables based on High Temperature Superconductor (HTS) possess the advantage of carrying high electrical current density $[1,2]$. HTS coated conductor coils are being used for superconducting power applications, such as superconducting fault current limiters [3, 4], and superconducting transformers [5, 6]. When HTS coils are operating in superconducting state, they are able to conduct higher amount of current but with much less loss than normal metal conductors. Moreover, for superconducting electrical machines and magnets, HTS coils can greatly increase the magnetic flux density, which leads to the improvement of overall efficiency and reductions in weight and size [7]. However, when HTS coils are operating with alternating current (AC), or in the presence of time varying magnetic field, they still sustain AC losses [8]. Therefore, it is necessarily crucial to investigate the AC loss characteristics of HTS coils as they are key components in superconducting applications.

Hysteresis AC loss is generally the most important loss in the study of superconductivity. The mechanism of hysteresis AC loss in Type-II superconductor can be described as: the vortices move to follow the changing magnetic field, where the pinning force represents an obstacle, and the accompanied power dissipation is called the hysteresis loss [8]. A transport current flowing through a superconductor generates a magnetic field around it, which is called the self-field. If with an $\mathrm{AC}$ transport current, the AC self-field also penetrates the superconductor during every cycle. Even if there is no external $\mathrm{AC}$ magnetic field, the variation of the self-field by $\mathrm{AC}$ transport current within the superconductor causes a hysteresis loss [9].

The circular HTS coil is one of the most common topologies used in many superconducting applications, such as superconducting transformer and magnetic resonance imaging (MRI) [10]. The round topology of a circular HTS coil offers HTS tapes better mechanical torsion when closely packed.

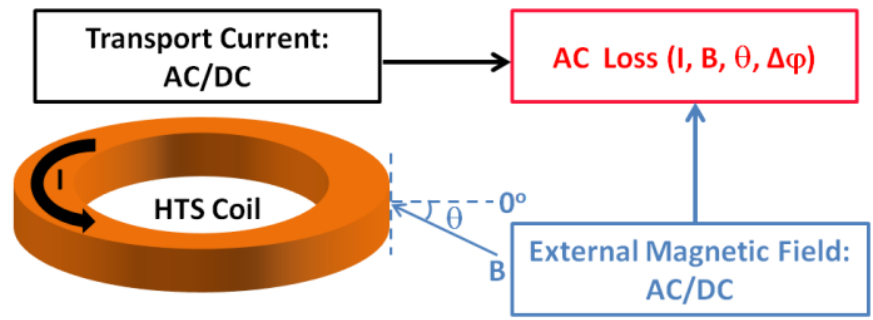

Figure 1. Factors affect AC loss on a HTS coil: current, field, angle, and phase difference. 


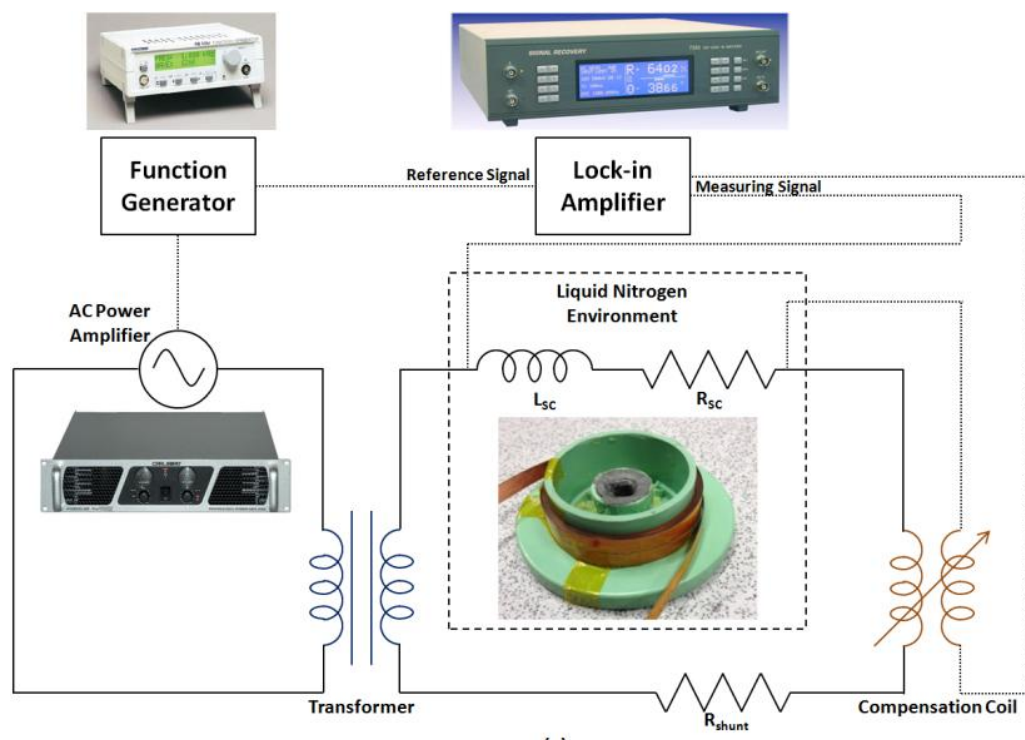

(a)

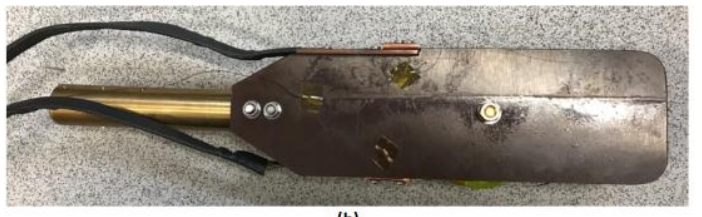

(b)

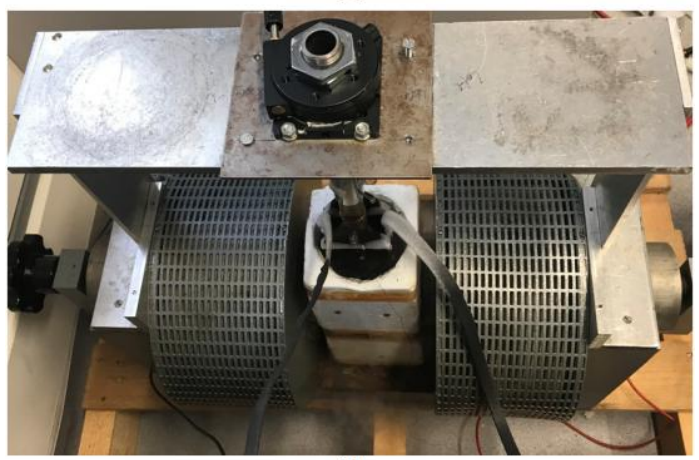

Figure 2. (a) Experimental schematic of AC loss measurements using electrical method, (b) Coil holder, (c) Electrometric magnet for field dependence analysis.

Furthermore, the axisymmetrical shape of circular HTS is easier to fabricate and model for the superconductivity research community. A thorough study of circular HTS coil is also beneficial for other topologies of HTS coil, e.g. racetrack coil, as its most critical part is at the end (round sharp curvature).

In the literature, there are some works on the AC loss measurement and simulation of HTS coils. Amemiya et al. have presented the AC loss from HTS tapes under external DC/AC magnetic fields [11]. Ciszek et al. analysed the angular dependence of AC transport losses in HTS tape on external DC magnetic fields [12]. Chiba et al. did research on the angular dependence of the AC loss from HTS stacks [13]. Nguyen et al. carried out AC loss measurement with a phase difference between the current and the applied magnetic field [14]. However, to the best of our knowledge, there is no literature demonstrates a comprehensive AC loss study with filed dependence on HTS coil under AC transport current and DC external magnetic field. Moreover, very few literatures are submitted to all the mentioned aspects at the same time: current, field, angle, and phase difference, shown in Figure 1. In this article, we present a comprehensive study on the AC losses in a circular HTS coated conductor coil, and demonstrate some new results and analysis. In order to validate some of the results, we have set up the experiment to measure the AC loss from a $2 \times 18$ circular double pancake coil using electrical method (shown in Figure 2 (a)). We have also built a 2D axisymmetric $\boldsymbol{H}$-formulation model using the FEM package COMSOL Multiphysics, which was used as primary tool for our investigation.

As shown in Figure 1, there are several scenarios that can cause AC losses in an HTS coil: Scenario 1 AC transport current and DC magnetic field, e.g. a superconducting inductive heater working on a magnetic material; Scenario 2 DC transport current and AC magnetic field, e.g. a DC superconducting magnet in MRI encountering various external AC signals; Scenario 3 AC transport current and AC magnetic
Table 1. Parameters for the circular HTS coil made by SuperPower SCS6050 (2012)

\begin{tabular}{ll}
\hline Parameters & Value \\
\hline Tape width & $6 \mathrm{~mm}$ \\
Superconducting layer thickness & $1 \mu \mathrm{m}$ \\
Tape total thickness & $100 \mu \mathrm{m}$ \\
KAPTON tape thickness & $100 \mu \mathrm{m}$ \\
Coil inner diameter & $5 \mathrm{~cm}$ \\
Coil total length & $6.3 \mathrm{~m}$ \\
Turn number & $2 \times 18$ \\
Tape Self-field $I_{\mathrm{c}}$ at $77 \mathrm{~K}$ & $115 \mathrm{~A}$ \\
Coil Self-field $I_{\mathrm{c}}$ at $77 \mathrm{~K}$ & $72 \mathrm{~A}$ \\
\hline
\end{tabular}

field, e.g. a fully superconducting electrical machine. Furthermore, as the HTS coated conductors have the anisotropic characteristics, the HTS coil under the magnetic field with different orientation angle $\theta$ (shown in Figure 1) should be studied for each case. For the scenario of AC transport current and $\mathrm{AC}$ magnetic field, the relative phase difference between AC current and AC field was analysed. To summarise, we carried out a current/field/angle/phase dependent AC loss $(\boldsymbol{I}, \boldsymbol{B}, \theta, \Delta \varphi)$ study of circular HTS coil, as follows.

For Scenario 1, both the experiment and simulation were carried out, and good agreement (average error 3.2\%) was seen in terms of AC loss magnitude, tendency, and angular dependence. For Scenario 2 and 3 the complexity of the measurement greatly increased (e.g. additional pick-up coil for magnetisation loss and further calibration system). Particularly for Scenario 3, both the transport current loss and magnetisation loss must be measured using separate experimental methods, and if along with the phase difference between the $\mathrm{AC}$ current and $\mathrm{AC}$ field, the measurement complexity will increase even further, and affects the measurement accuracy. There are a few successful studies of numerical loss calculation using $\boldsymbol{H}$-formulation for HTS under complex AC and DC conditions, e.g. numerical analysis of $\mathrm{AC}$ loss in YBCO coated conductor tapes carrying DC and AC offset transport current [15], and ripple field losses in 

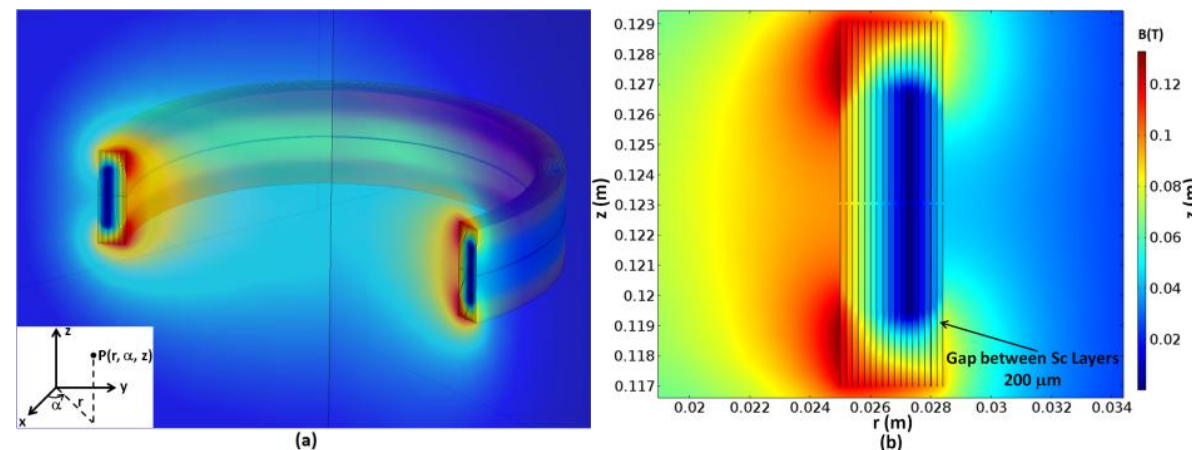

(b)

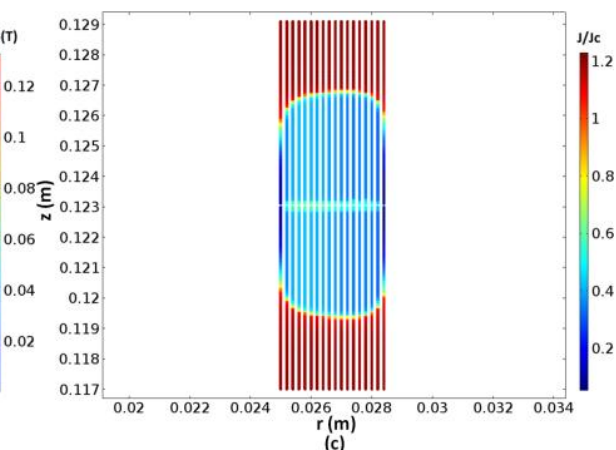

(c)

Figure 3. (a) 3D and (b) 2D magnetic flux density of the circular double pancake coil with an AC current of $60 \mathrm{~A}$ (peak point); (c) normalised current density ratio $\left(J / J_{c}\right)$ of the circular double pancake coil with an AC current of $60 \mathrm{~A}$ (peak point), where the cross-sections of tape have been zoomed up 100 times for better visualisation.

direct current biased superconducting tapes [16]. Therefore, a powerful and do-it-all numerical model using $\boldsymbol{H}$-formulation for the HTS coil is necessary to be established, which could efficiently calculate the AC loss from Scenario 2 and 3, and other complex conditions.

\section{Experiment Set-up}

\subsection{Fabrication of the circular HTS coil}

The circular HTS coil used in this experiment was fabricated from $6 \mathrm{~mm}$ wide SuperPower SCS6050 tape (manufactured around 2012). The critical current $I_{c}$ of a single tape in its self-field was measured to be $115.3 \mathrm{~A}$. The total length of the tape for winding the coil was $6.3 \mathrm{~m}$, whose surface was insulated by KAPTON tapes. As shown in Figure 2 (a), the configuration of coil was a double circular pancake, with $2 \times 18$ turns. The critical current $I_{c}$ of the coil in its self-field was measured to be $72.1 \mathrm{~A}$. More details of the circular HTS coil are in Table 1.

\subsection{AC loss measurement}

Figure 2(a) presents the schematic for the measurement of the AC losses in a circular HTS coil using the electrical method. The function generator (digimess ${ }^{\circledR}$ FG100) produced timevarying sinusoidal signals as the reference input of the lock-in amplifier (Signal Recovery 7265), and this AC signal was also amplified by a power source (Carlsbro Powerline Pro 1200) in the primary circuit side. In secondary circuit, the AC current was raised 16 times by using a step-down transformer. As shown in Figure 2(b), the HTS coil was fixed by a coil holder, and immersed into a liquid nitrogen environment at $77 \mathrm{~K}$. The HTS coil was located in the presence of uniform magnetic field generated by the iron magnet (shown in Figure 2(c)). The coil holder was rotatable, and we could rotate the coil. Therefore, we were able to change the orientation of magnetic field angle $\theta$ (shown in Figure 1) to the HTS tape surface of the coil. The magnitude of AC current in the transformer secondary side was obtained using the voltage across the shunt resistor (shown in Figure 2(a)) divided by its value of resistance, and monitored by a high accuracy data acquisition card linked to the PC with the software NI SignalExpress.

Obtaining the resistive voltage component (in-phase voltage component) is a key step for AC loss measurement of HTS coil. Actually, there were two possible methods to get the in- phase voltage component. (a) We extracted a resistive voltage component from the shunt resistor in the circuit as the reference signal, and then we used the lock-in amplifier to pick up the in-phase voltage component from superconducting tape for AC loss measurement; (b) We used the function generator signal as both the source for AC system and the reference signal for lock-in amplifier, and then we used the adjustable compensation coil to compensate the inductive voltage quantity (to get the minimum voltage) in the measuring signal side, which enables the lock-in Amplifier to extract the voltage in-phase with the current of HTS tape. We tested both method (a) and (b), which gave us the same results. We used the method (b) to carry out all the measurement. The transport AC loss can be calculated as [17]:

$$
Q_{a c_{-} l o s s}=\frac{I_{r m s} \cdot V_{r m s}}{f}
$$

where $I_{r m s}$ is the AC transport current flowing through the HTS tape, $V_{r m s}$ is the in-phase voltage with current $I_{r m s}$, and $f$ is the frequency of the AC current.

\section{Simulation Method}

\section{1 $2 \mathrm{D}$ axisymmetric $\boldsymbol{H}$-formulation}

In order to model the AC losses from the circular HTS coil, we used the 2D axisymmetric $\boldsymbol{H}$-formulation as the suitable FEM method [18-20]. An example of our double circular pancake coil using 2D axisymmetric $\boldsymbol{H}$-formulation is presented in Figure 3: (a) 3D and (b) 2D magnetic flux density of the circular double pancake coil with an AC current of $60 \mathrm{~A}$ (peak point); (c) normalised current density ratio $\left(J / J_{c}\right)$ of the circular double pancake coil with an AC current 60 A (peak point) where the cross-sections of the tape have been zoomed up 100 times for better visualisation. The distribution of the current density has been artificially expanded because otherwise it would not be visible on this scale. A 2D axisymmetric model of the coil matches the experimental situation only in the case of self-field or when the external magnetic field is applied parallel to the axis of the coil (axis z in figure $3 \mathrm{a}$, theta $=90$ degrees in figure 1). In the other situations with external magnetic field, the axial symmetry is broken and a 3D model would be necessary. However, a full 3D model of the coil is computationally too demanding. For this reason, we kept the simplifying assumption of a $2 \mathrm{D}$ axisymmetric model, because with the exception theta $=90$ 
Table 2. Parameters for the modelling of the circular HTS coil

\begin{tabular}{ll}
\hline Parameters & Value \\
\hline Tape width & $6 \mathrm{~mm}$ \\
Superconducting layer thickness & $1 \mu \mathrm{m}$ \\
$\mu_{0}$ & $4 \pi \times 10^{-7} \mathrm{H} / \mathrm{m}$ \\
$n$ (E-J Power Law index) & 25 \\
$J_{c 0}$ & $2.1 \times 10^{10} \mathrm{~A} / \mathrm{m}^{2}$ \\
$E_{0}$ & $10^{-4} \mathrm{~V} / \mathrm{m}$ \\
$B_{c}$ & $35 \mathrm{mT}$ \\
$k$ & 0.25 \\
$b$ & 0.6 \\
$f$ & $50 \mathrm{~Hz}$ \\
\hline
\end{tabular}

degrees, for which the 2D axisymmetric model is appropriate. It implies that there is always a magnetic field component perpendicular to the tape for the whole length of the coil. This represents a kind of worst case scenario and provides a reasonable upper limit for the losses. The $\boldsymbol{H}$-formulation was used successfully to model the losses from HTS under the action of AC current and AC magnetic field [19-21]. General $\boldsymbol{H}$-formulation consists of Ohm's Law (2), Ampere's Law (3), Faraday's Law (4), constitutive Law (5), and E-J power Law (6):

$$
\begin{gathered}
\boldsymbol{E}=\rho \boldsymbol{J} \\
\nabla \times \boldsymbol{H}=\boldsymbol{J} \\
\nabla \times \boldsymbol{E}=-\frac{\partial \boldsymbol{B}}{\partial t} \\
\boldsymbol{B}=\mu_{0} \mu_{r} \boldsymbol{H} \\
E=E_{0}\left(\frac{J}{J_{c}}\right)^{n}
\end{gathered}
$$

Where $\boldsymbol{E}$ is the electric field, $\rho$ is the resistivity, $\boldsymbol{J}$ is the current density, $\boldsymbol{H}$ is the magnetic field intensity, $\boldsymbol{B}$ is the magnetic flux density, $\mu_{0}$ is the permeability of free space, $\mu_{r}$ is the relative permeability. Equation (6) presents the $E-J$ power law of HTS formulation, where $E_{0}$ is the characteristic electric field, $J_{c}$ is the critical current density and $n$ is the $\mathrm{n}$ index. By merging equation (2), (3), (4), (5) and (6), the general form of partial differential equation (PDE) for variables $\boldsymbol{H}$ to be computed by COMSOL Multiphysics is [22]:

$$
\frac{\partial\left(\mu_{0} \mu_{r} \boldsymbol{H}\right)}{\partial t}+\nabla \times(\rho \nabla \times \boldsymbol{H})=0
$$

For the 2D axisymmetric $\boldsymbol{H}$-formulation, we used the cylindrical coordinates $(r, \theta, z)$, and the governing equations should be modified as:

$$
\begin{gathered}
-\frac{\partial \boldsymbol{E}_{\theta}}{\partial z}=-\frac{\partial\left(\mu_{0} \mu_{r} \boldsymbol{H}_{r}\right)}{\partial t} \\
\frac{\boldsymbol{E}_{\theta}}{r}+\frac{\partial \boldsymbol{E}_{\theta}}{\partial r}=-\frac{\partial\left(\mu_{0} \mu_{r} \boldsymbol{H}_{z}\right)}{\partial t}
\end{gathered}
$$

\subsection{AC loss calculation}

In the FEM model, we used the real dimension of the SuperPower SCS6050 tape, with a superconducting layer 1 $\mu \mathrm{m}$ thick. The geometry of modelling a $2 \times 18$ turns double circular pancake coil was exactly the same as the real experimental coil described above, and this method was in order to achieve better consistency. The $E$-J Power Law factor $n$ used for modelling was 25 . This is a moderate value when a single tape is in the presence of DC magnetic field between $0-500 \mathrm{mT}$, and according to our measurements the $n$ variation of the SCS6050 tape is not significant within this field range. For the modelling of HTS coil by COMSOL, an anisotropic $\boldsymbol{B}$ dependent critical current model was implanted [23]:

$$
J_{c}(B)=\frac{J_{c 0}}{\left(1+\frac{\sqrt{\left(k \boldsymbol{B}_{\text {para }}\right)^{2}+\boldsymbol{B}_{\text {perp }}^{2}}}{\boldsymbol{B}_{c}}\right)^{b}}
$$

where $J_{c 0}=2.1 \times 10^{10} \mathrm{~A} / \mathrm{m}^{2}, k=0.25, B_{c}=0.3$, and $b=0.6$. Other relevant simulation parameters are listed in Table 2 . The critical current of tape and coil were calculated with the same $\boldsymbol{H}$-formulation model using a slow current ramp. The calculated the critical current of the single tape in self-field was $114.5 \mathrm{~A}$, and the critical current of the double pancake coil was 71.6 A. These two critical current values are very close to the experimental results (single tape $I_{c}=115.3 \mathrm{~A}$, and coil $I_{c}=72.1 \mathrm{~A}$ ) mentioned above, which proves a good consistency between modelling and experiment.

It is known that there are four main sources of $\mathrm{AC}$ losses in Type-II hard superconductors, which are hysteresis AC losses, ferromagnetic AC losses, eddy-current AC losses and coupling AC losses [8]. In our study, there were no ferromagnetic losses as the SuperPower SCS6050 tape uses non-magnetic substrate. The simulations were carried out using a relatively low frequency of the $\mathrm{AC}$ power system current at $50 \mathrm{~Hz}$, and the small amount of eddy-current $\mathrm{AC}$ losses in the metal layers and the substrate can be negligible $[24,25]$. Similar to eddy-current losses, the coupling loss can be negligible comparing to hysteresis losses when operating in power frequencies [8]. Therefore, hysteresis AC losses dominate in all the cases in this study.

In the model, the transport current was injected into the HTS tapes using the Global constraint from general PDE Physics, a module from COMSOL [20]. The value of the transport current $I_{t}$ was computed by the integration of the current density $\boldsymbol{J}$ on the superconducting cross-section $\Omega$ :

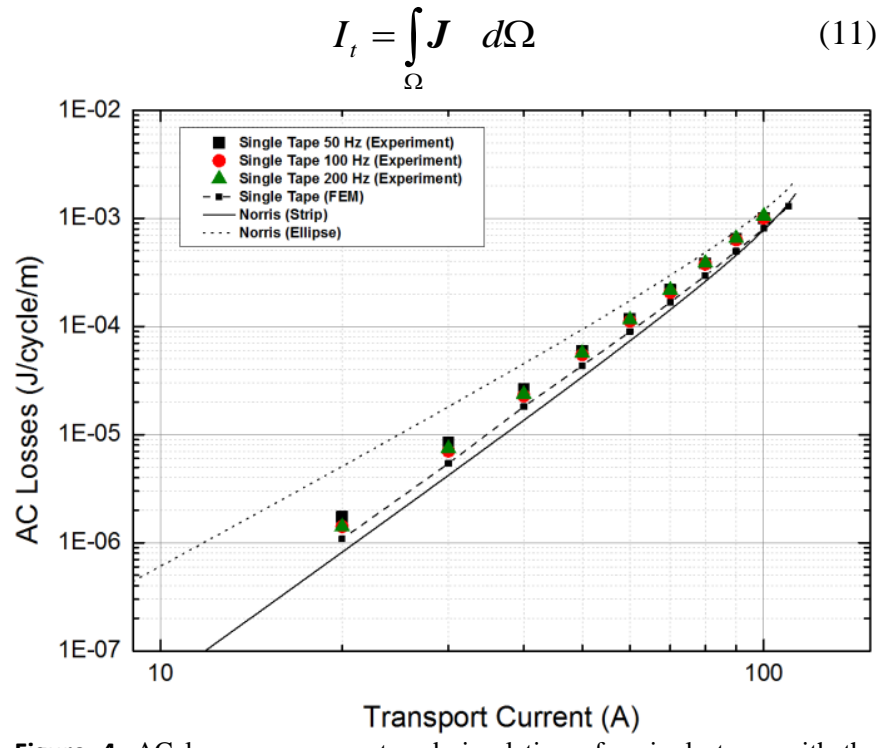

Figure 4. AC loss measurement and simulation of a single tape, with the references of Norris strip and Norris ellipse. 


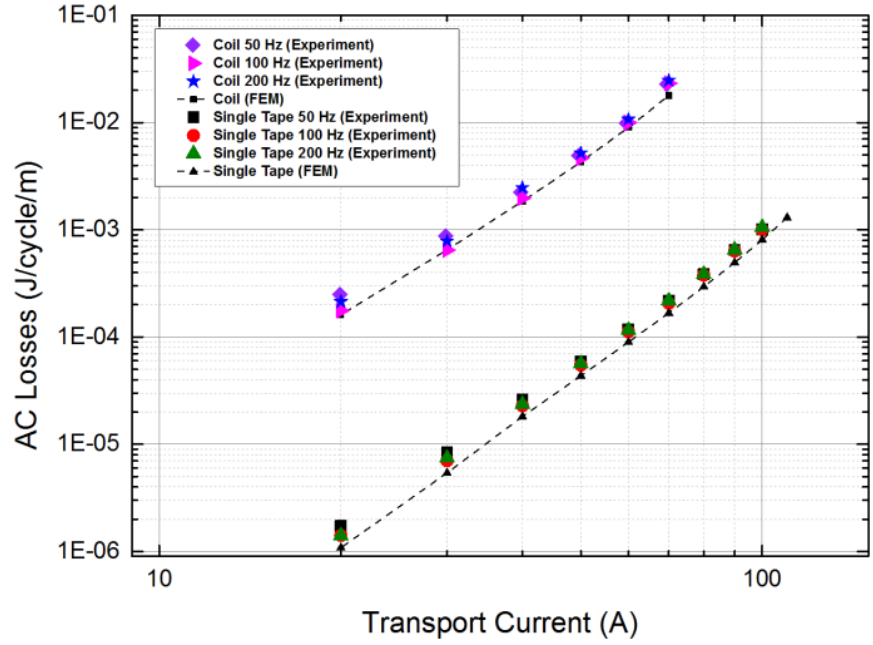

Figure 5. Comparison of HTS coil and single tape: AC loss measurement and simulation.

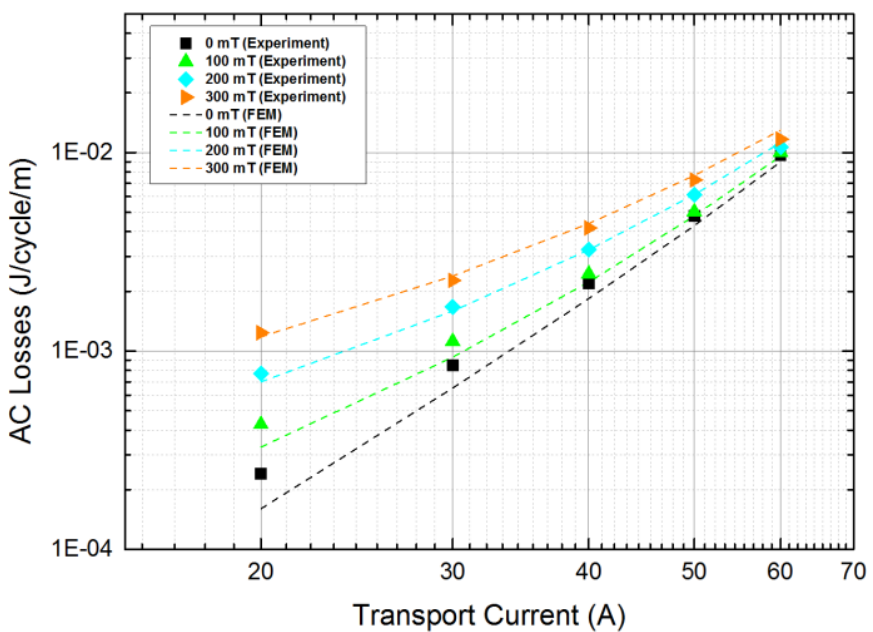

Figure 6. AC loss measurement and simulation of the HTS coil, with AC transport current and DC external magnetic field perpendicular to the HTS tape surface of the coil.

The AC loss of the domain was calculated using the power density $(\boldsymbol{E} \cdot \boldsymbol{J})$ integration [26]:

$$
Q=\frac{2}{T} \int_{0.5 T}^{T} \int_{\Omega} \boldsymbol{E} \cdot \boldsymbol{J} d \Omega d t
$$

where $\mathrm{T}$ is the period of cycle and $\Omega$ is the domain of interest.

\section{Results: AC Transport Current and External DC Magnetic Field}

\subsection{Basic frequency dependence test of AC loss for the HTS tape and coil}

We started with the simplest situation: AC loss measurement and simulation of a single tape. The AC loss measurement was carried out using frequency $50 \mathrm{~Hz}, 100 \mathrm{~Hz}$ and $200 \mathrm{~Hz}$, and transport current from $20 \mathrm{~A}$ to $100 \mathrm{~A}$. The FEM simulation of a single tape was also performed with $\mathrm{AC}$ transport current from $20 \mathrm{~A}$ to $100 \mathrm{~A}$ at $50 \mathrm{~Hz}$. In Figure 4, the measurement results present the $\mathrm{AC}$ losses per cycle of a single tape were frequency independent within the range of 50 to $200 \mathrm{~Hz}$. As shown in Figure 4, both the AC losses from the experiment

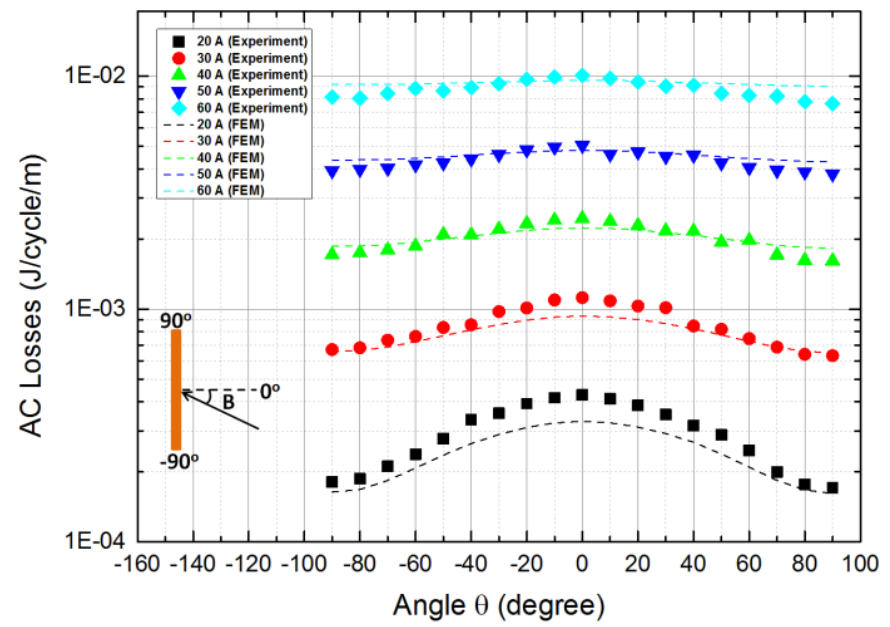

Figure 7. AC loss measurement and simulation of the HTS coil, with increasing AC transport current and angular dependence of external DC magnetic field $100 \mathrm{mT}$.

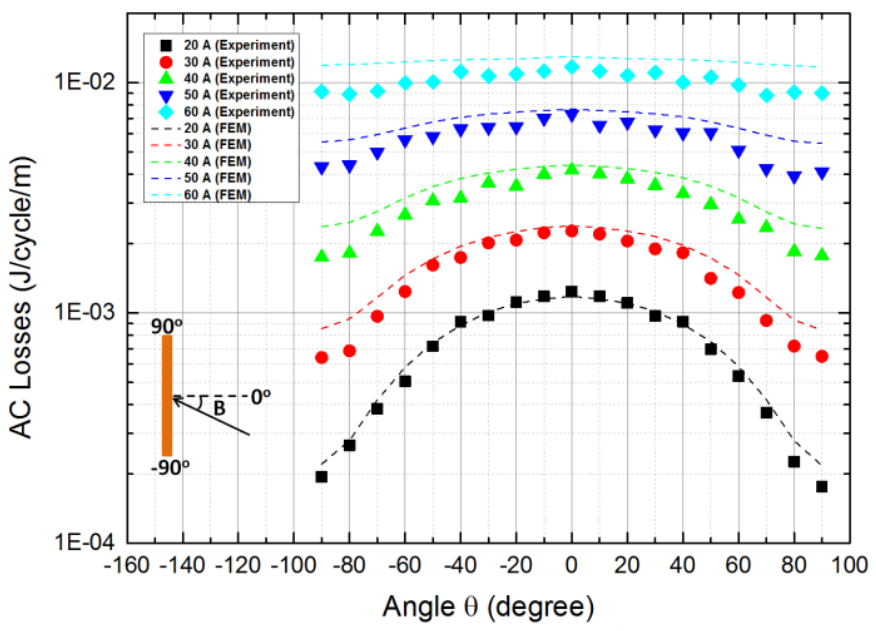

Figure 8. AC loss measurement and simulation of the HTS coil, with increasing AC transport current and angular dependence of external DC magnetic field $300 \mathrm{mT}$

and simulation were within the range of the Norris strip and Norris ellipse [27], and the experimental results agreed well with the simulation results.

Similarly, the AC losses of the circular double pancake HTS coil were measured with frequency $50 \mathrm{~Hz}, 100 \mathrm{~Hz}$ and $200 \mathrm{~Hz}$, and transport current from $20 \mathrm{~A}$ to $70 \mathrm{~A}$ and the FEM calculation of that coil tape was also performed using the same $\mathrm{AC}$ transport current at $50 \mathrm{~Hz}$. As presented in Figure 5, the $\mathrm{AC}$ losses of the coil were frequency-independent within the range of 50 to $200 \mathrm{~Hz}$, similar to single tape. For both the tape and coil, the experimental results were consistent with the simulation results. It can be seen from Figure 5 that the AC losses from coil were approximately 2 orders of magnitude higher than the AC losses from the single tape. This is due to the magnetic interaction between coil turns, as previously reported [28].

\subsection{AC loss and its angular dependency analysis with AC transport current and external DC magnetic field}

Figure 6 presents the AC loss measurement and simulation of the HTS coil, with AC transport current and DC external magnetic field perpendicular to the HTS tape surface of the coil. From Figure 7, it can be seen from both the experiment 
and simulation that the difference in AC loss with each set of external DC field became smaller when the transport current increased, e.g. from the experiment the loss ratio of $\mathrm{Q}_{3} 300 \mathrm{mT} / \mathrm{Q}_{-0 \mathrm{mT}}$ was 5.1 with $20 \mathrm{~A}$ AC transport current, then decreased to 1.2 when the $\mathrm{AC}$ transport current increased to $60 \mathrm{~A}$. this phenomenon is similar to that reported in [11]. Overall, the same trend was observed in both experiment and simulation.

Figure 7 illustrates the $\mathrm{AC}$ loss measurement and simulation of the HTS coil, with increasing AC transport current and angular dependence of the external DC magnetic field of $100 \mathrm{mT}$, while Figure 8 presents the same content with the external DC magnetic field of $300 \mathrm{mT}$ for comparison. Angle $\theta$ refers to the external magnetic field with different orientation to the HTS tape surface of the coil (e.g. as shown in Figure 1, $\theta$ changing from -90 degree to +90 degree, means, magnetic field orientation to the HTS tape surface of the coil changing, from parallel, to perpendicular, then to parallel again). We increased the $\mathrm{AC}$ transport current from $20 \mathrm{~A}$ to $60 \mathrm{~A}$, and DC external magnetic from 100 to $300 \mathrm{mT}$. We also changed the angle $\theta$ from -90 degree to 90 degree. For both the $100 \mathrm{mT}$ and $300 \mathrm{mT}$ cases, the angular dependence with a smaller AC transport current was more obvious than the angular dependence with a larger AC transport current, e.g. from the experiment with $300 \mathrm{mT}$ DC field, the loss ratio $\mathrm{Q}_{\text {_odeg }} / \mathrm{Q}_{-90 \mathrm{deg}}$ was 7.0 with an $\mathrm{AC}$ transport current of $20 \mathrm{~A}$, while the loss ratio $\mathrm{Q}_{\text {_odeg }} / \mathrm{Q}_{9} 90 \mathrm{deg}$ was 1.29 with an $\mathrm{AC}$ transport current of $60 \mathrm{~A}$. This is due to the fact that, when a relatively large transport AC current flows in the coil, the external DC field is less influential on the reduction of the critical current. In general, for both Figure 7-8, the agreement between the experiment and the simulation is good (average error 3.2\%). However, there was slight discrepancy between experiment and simulation (low transport current in Figure 7, and high current in Figure 8), which are probably due to local effects (e.g. uniformity of $J_{c}$ near the edges, alignment of the tapes) was not included in the model.

Figure 9 shows the AC loss measurement and simulation of the HTS coil, with the same AC transport current of $30 \mathrm{~A}$ and angular dependence of the external DC magnetic field increasing from $100 \mathrm{mT}, 200 \mathrm{mT}$ to $300 \mathrm{mT}$, while Figure 10 presents the same content with an $\mathrm{AC}$ transport current of 60 A for comparison. As shown in Figure 9, for a transport

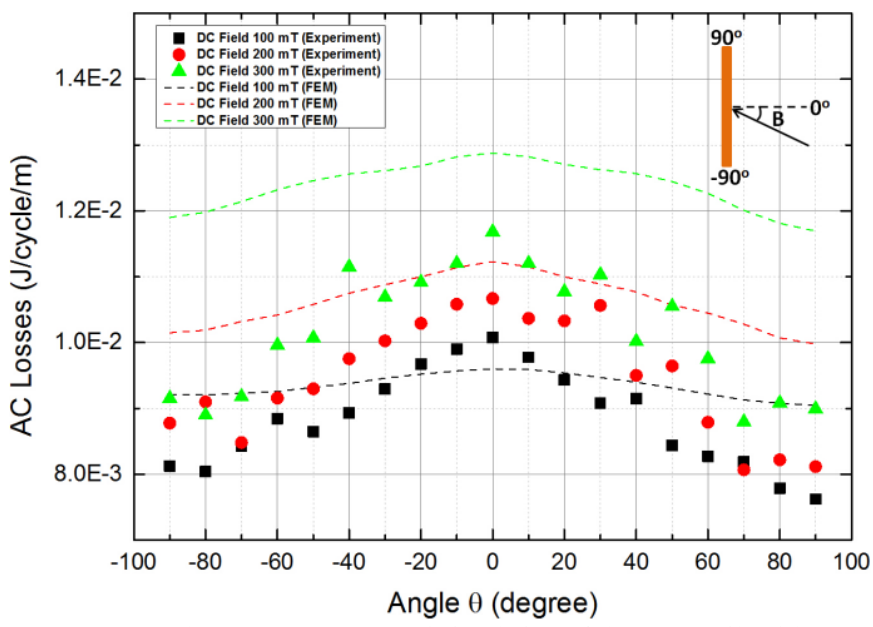

Figure 10. AC loss measurement and simulation of the HTS coil, with same $\mathrm{AC}$ transport current $60 \mathrm{~A}$ and angular dependence of external DC magnetic field increasing from $100 \mathrm{mT}, 200 \mathrm{mT}$ to $300 \mathrm{mT}$.

current of $30 \mathrm{~A}$, the $\mathrm{AC}$ loss was more angular dependent with a stronger external DC magnetic field, e.g. from the experiment, the loss ratio $\mathrm{Q}_{0 \text { odeg }} / \mathrm{Q}_{-90 \mathrm{deg}}$ was 1.8 with an external DC field of $100 \mathrm{mT}$, whilst $\mathrm{Q}_{-}$odeg $/ \mathrm{Q}_{-} 90 \mathrm{deg}$ was 3.5 with an external DC field of $300 \mathrm{mT}$. By contrast, as shown in Figure 10, for a higher transport current of $60 \mathrm{~A}$, the AC loss was no longer angular dependent as the intensity of the external DC magnetic field increased, e.g. the loss ratio Q_odeg $/ Q_{-90 d e g}$ with an external DC field of $100 \mathrm{mT}$ was very close to $\mathrm{Q}_{-}$odeg $/ \mathrm{Q}_{-} 90 \mathrm{deg}$ with an external DC field of $300 \mathrm{mT}$. From Figure 9 and Figure 10 one can note that the simulation results gradually exceeded the experimental results with the increasing background magnetic field. A possible reason for this could be that (10) does not perfectly represent the tape's behaviour at high fields. Despite this, the model is able to produce the general magnitudes and tendencies of the experiments.

\section{Results: DC Transport Current and External AC}

\section{Magnetic Field}

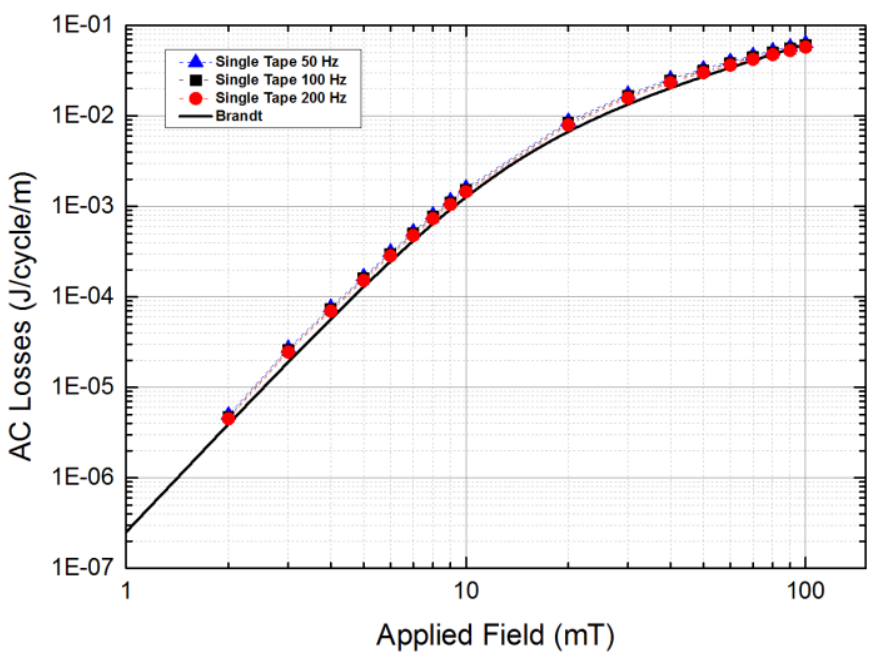

Figure 11. AC loss simulation of the single tape in the presence of external AC magnetic field with frequencies of $50 \mathrm{~Hz}, 100 \mathrm{~Hz}$ and $200 \mathrm{~Hz}$, with the reference of Brandt curve. 


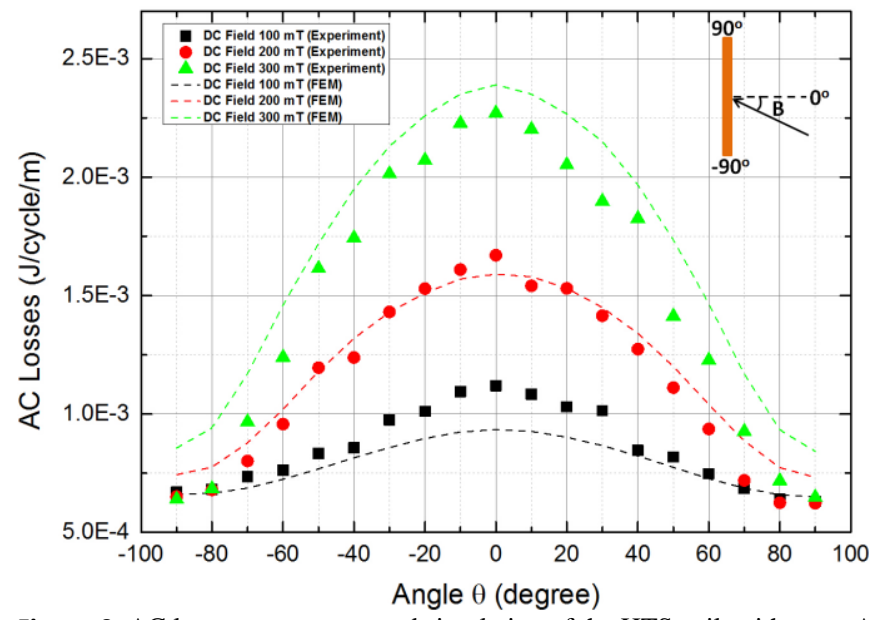

Figure 9. AC loss measurement and simulation of the HTS coil, with same AC transport current $30 \mathrm{~A}$ and angular dependence of external DC magnetic field increasing from $100 \mathrm{mT}, 200 \mathrm{mT}$ to $300 \mathrm{mT}$.

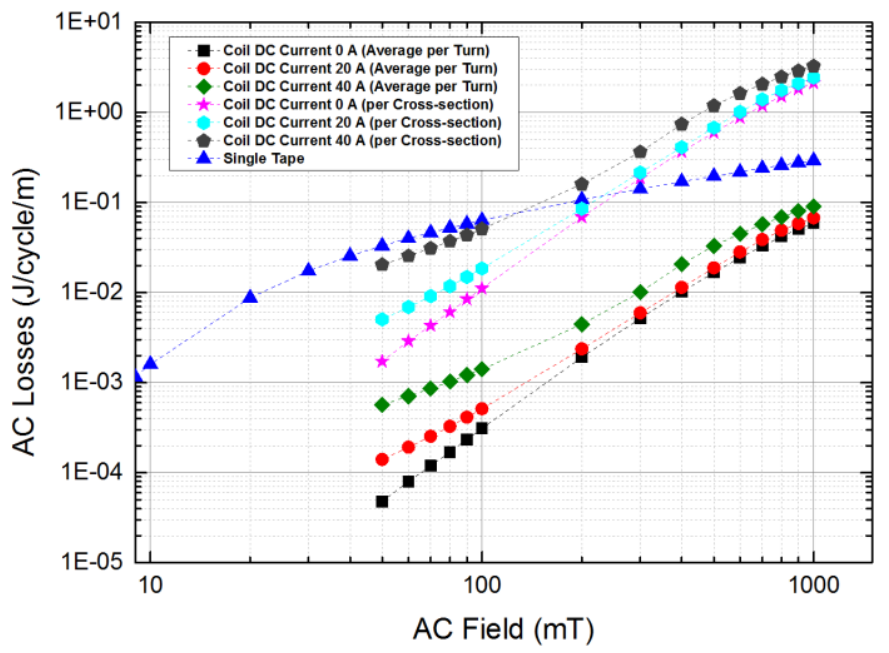

Figure 12. AC loss simulation of the coil with different $D C$ transport currents $(0 \mathrm{~A}, 20 \mathrm{~A}$, and $40 \mathrm{~A})$ and increasing external $\mathrm{AC}$ magnetic field, with the reference of AC loss from a single tape.

As we have verified the capability of the FEM coil model in Section 4, achieving good agreement with experimental results in terms of AC loss magnitude, tendency, and angular dependence, we believe that FEM model is able to produce convincing results for the following conditions: the simultaneous presence of $\mathrm{DC}$ transport current and $\mathrm{AC}$ magnetic field in Section 5, and of AC transport current and AC magnetic field later in Section 6.

\subsection{Basic test of AC loss for HTS tape and coil with DC transport current and external AC magnetic field}

We started to simulate the single SCS6050 tape in the presence of AC magnetic field to test the basic consistency. The AC loss of a single SCS6050 tape was calculated under the AC magnetic field with three different frequencies $50 \mathrm{~Hz}$, $100 \mathrm{~Hz}$, and $200 \mathrm{~Hz}$. As shown in Figure 11, the AC loss per cycle of the single tape was frequency-independent, and the loss trend well matched the Brandt curve.

As presented in Figure 12, the AC loss simulation of the coil with different DC transport current $(0 \mathrm{~A}, 20 \mathrm{~A}$, and $40 \mathrm{~A})$ and increasing external AC magnetic field was carried out, with the reference of $\mathrm{AC}$ loss from a single tape in the presence of the same external AC magnetic field. Figure 12

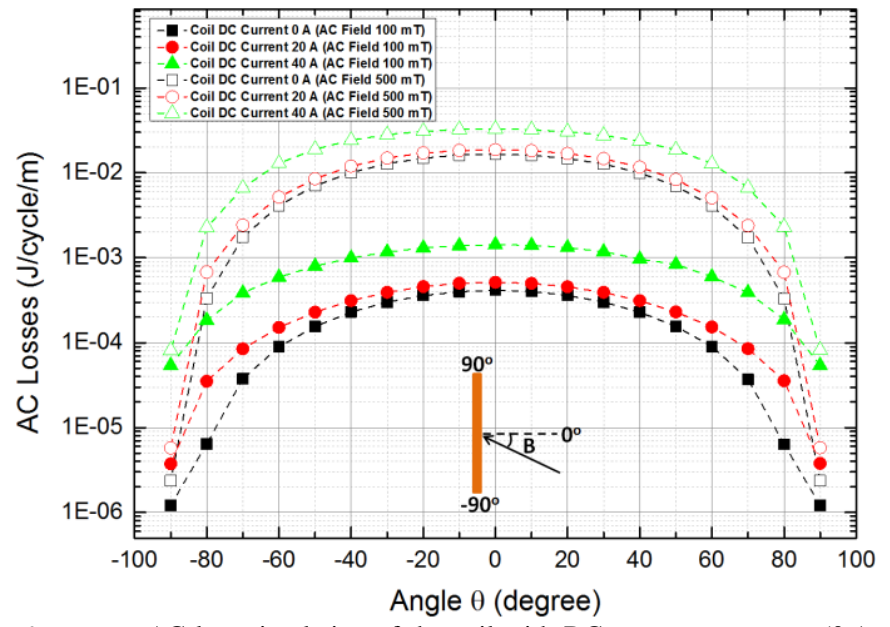

Figure 13. AC loss simulation of the coil with DC transport currents $(0 \mathrm{~A}$, $20 \mathrm{~A}$, and $40 \mathrm{~A}$ ) and the angular dependence of the external $\mathrm{AC}$ magnetic field at $100 \mathrm{mT}$ and $500 \mathrm{mT}$.

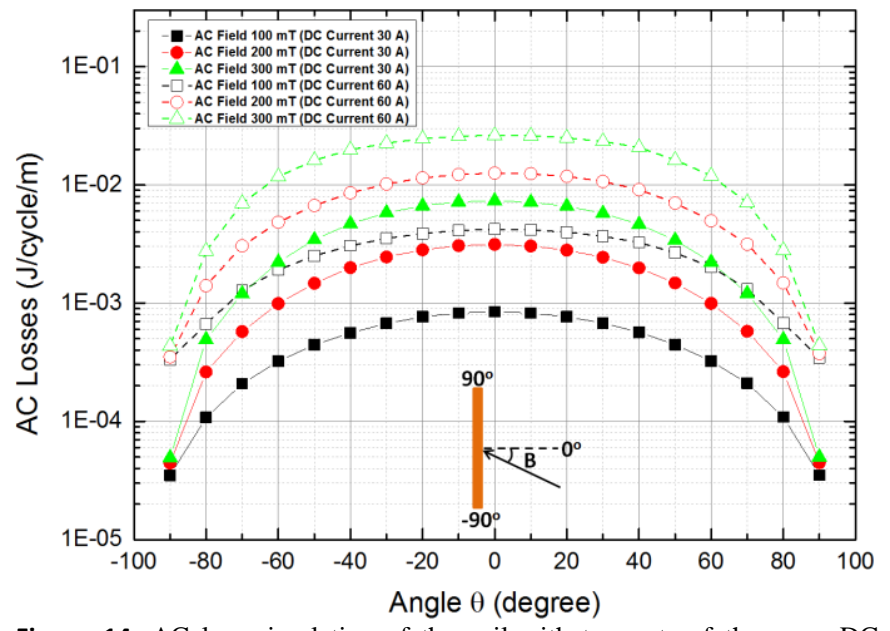

Figure 14. AC loss simulation of the coil with two sets of the same DC transport currents $(30 \mathrm{~A}$ and $60 \mathrm{~A})$ and the angular dependence of increasing the external AC magnetic field (100 mT, $200 \mathrm{mT}$, and $300 \mathrm{mT}$ ).

shows that, in the coil, the average $\mathrm{AC}$ loss per turn was lower than the AC loss of the single tape, and even the AC loss per cross-section (per turn multiply by $2 \times 18$ turn) was still lower than the $\mathrm{AC}$ loss of the single tape with an external AC magnetic field below $200 \mathrm{mT}$. This is because of the shielding effect from each turn of the HTS coil. However, this effect became weaker with an increasing external $\mathrm{AC}$ magnetic field: the increasing rate of loss in coil was faster than the increasing rate of loss in the single tape, and the average coil $\mathrm{AC}$ loss per turn had the trend to surpass the AC loss of the single tape.

In principle, in the presence of the same AC magnetic field, the magnetisation loss of a superconducting tape with an increasing DC transport current should not change (if the tape is not fully penetrated). However, it should be considered that the DC transport current within the HTS coil generated the self-field which could decrease the critical current of the coil, thus increasing the AC loss of the coil. That phenomenon occurred in Figure 12, and it can be observed that the difference of coil AC loss between the DC transport currents of $20 \mathrm{~A}$ and $40 \mathrm{~A}$, was greater than that of DC transport currents of $0 \mathrm{~A}$ and $20 \mathrm{~A}$, which could be because the critical current reduction between DC currents of $20 \mathrm{~A}$ and $40 \mathrm{~A}$ is more significant than DC currents between $0 \mathrm{~A}$ and $20 \mathrm{~A}$. 


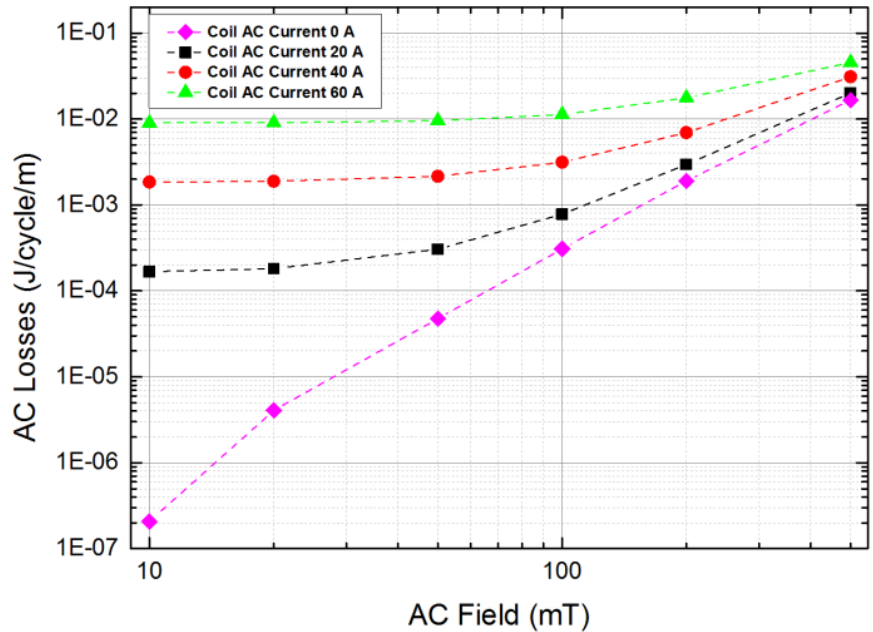

Figure 15. AC loss simulation of the HTS coil, with AC transport current and external AC magnetic field perpendicular to the HTS tape surface of the coil.

\subsection{AC loss and its angular dependency analysis with DC transport current and external AC magnetic field}

Figure 13 shows the AC loss simulation of the coil with DC transport currents $(0 \mathrm{~A}, 20 \mathrm{~A}$, and $40 \mathrm{~A})$ and angular dependence of external $\mathrm{AC}$ magnetic field at $100 \mathrm{mT}$ and $500 \mathrm{mT}$. The angular dependency with a lower DC transport current was stronger than that with higher transport current. For example, from the simulation with a $100 \mathrm{mT}$ AC field, the loss ratio of $\mathrm{Q}_{-0 \mathrm{deg}} / \mathrm{Q}_{-90 \mathrm{deg}}$ was 342.5 with an $\mathrm{DC}$ transport current of $0 \mathrm{~A}$, whilst the loss ratio of $\mathrm{Q}_{-}$ded $/ \mathrm{Q}_{-} 90 \mathrm{deg}$ was 26.4 with DC transport current of $40 \mathrm{~A}$. From the comparison of two cases of AC fields at $100 \mathrm{mT}$ and $500 \mathrm{mT}$, it can be seen that the angular dependence was more apparent with the larger external AC field, e.g. from the simulation with a $20 \mathrm{~A} \mathrm{DC}$ transport current, the loss ratio of $\mathrm{Q}_{-} 0 \mathrm{deg} / \mathrm{Q}_{-} 90 \mathrm{deg}$ was 136.6 with an AC magnetic field $100 \mathrm{mT}$, while the loss ratio of Q_odeg $/ \mathrm{Q}_{90 \text { deg }}$ was 3245.7 with an AC magnetic field $500 \mathrm{mT}$. These two phenomena above are similar to Scenario 1 AC transport current \& DC external magnetic field. However, the effect of increasing the DC transport current with a given (fixed) $\mathrm{AC}$ external field is different from increasing the $\mathrm{AC}$ transport current with a given (fixed) DC external field. This is because the AC loss was substantially caused by two different sources, the AC transport current and the AC external magnetic field, which have different magnetic field penetration patterns.

Figure 14 depicts the AC loss of the coil for two sets of DC transport currents (30 and $60 \mathrm{~A}$ ) and the angular dependence of increasing the external AC magnetic field (100 mT, $200 \mathrm{mT}$ and $300 \mathrm{mT}$ ). For the same DC transport current, the angular dependence was more evident with an increasing external AC magnetic field. By comparing the two sets of transport currents, $30 \mathrm{~A}$ and $60 \mathrm{~A}$, the angular dependence did not change too much, which can be seen from the fact that the loss curves of $30 \mathrm{~A}$ and $60 \mathrm{~A}$ were almost parallel. This phenomenon is different from Scenario 1, where the angular dependence with a higher transport current was much smaller than that with a lower transport current. The reason of this difference can be that the origins of loss were different. The losses in Scenario 2 were entirely from the AC magnetic field,

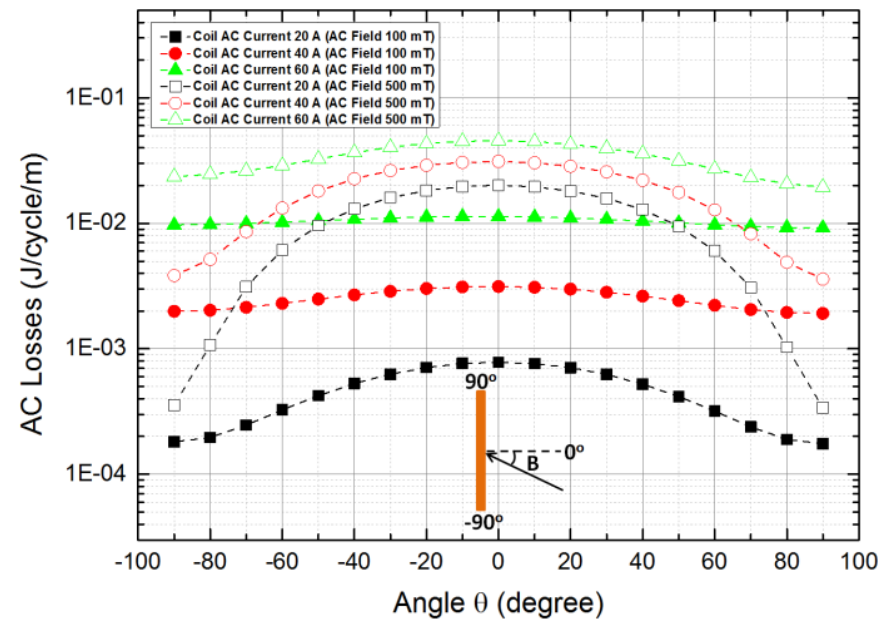

Figure 16. AC loss simulation of the coil, with AC transport currents $(20 \mathrm{~A}$, $40 \mathrm{~A}$, and $60 \mathrm{~A}$ ) and the angular dependence of the external $\mathrm{AC}$ magnetic field (100 $\mathrm{mT}$ and $500 \mathrm{mT})$.

and the magnitude of $\mathrm{AC}$ magnetic field directly affected the AC losses in HTS coil. Therefore it is reasonable that the AC loss angular dependence with higher magnitude of magnetic field in Scenario 2 was stronger than that from Scenario 1.

\section{Results: AC Transport Current and External AC}

\section{Magnetic Field}

In this section, the coil AC loss was calculated under the action of AC transport current and external AC magnetic field, and its angular dependency was compared to the experimental and simulation results under the action of AC transport current and external DC magnetic field. We have also investigated the effect of phase difference $\Delta \varphi$ between the AC transport current and the external AC magnetic field on the total AC loss of the coil.

\subsection{AC losses in HTS coil with AC transport current and external AC magnetic field perpendicular to the HTS tape surface of the coil}

Figure 15 presents the AC loss simulation of the HTS coil, with $\mathrm{AC}$ transport current and external $\mathrm{AC}$ magnetic field perpendicular to the HTS tape surface of the coil. The AC loss increasing rate of $0 \mathrm{~A}$ transport current started with a steep slope but the rate decreased with the increasing external AC magnetic field. By contrast, the other three AC loss curves for the AC transport currents of $20 \mathrm{~A}, 40 \mathrm{~A}$ and $60 \mathrm{~A}$, started with mild slopes but later gradually increased with the increasing external AC magnetic field. Eventually, these four loss curves for $\mathrm{AC}$ transport currents of $0 \mathrm{~A}, 20 \mathrm{~A}, 40 \mathrm{~A}$ and $60 \mathrm{~A}$ moved closer when the external AC magnetic field approached a high value. This tendency is consistent with the work in literature [11].

\subsection{AC loss and its angular dependency analysis with $A C$ transport current and external AC magnetic field}

Figure 16 illustrates the AC loss simulation of the coil, with $\mathrm{AC}$ transport currents $(20 \mathrm{~A}, 40 \mathrm{~A}$, and $60 \mathrm{~A})$ and the angular dependence of the external AC magnetic fields of $100 \mathrm{mT}$ and $500 \mathrm{mT}$. The angular dependency with a lower AC transport 


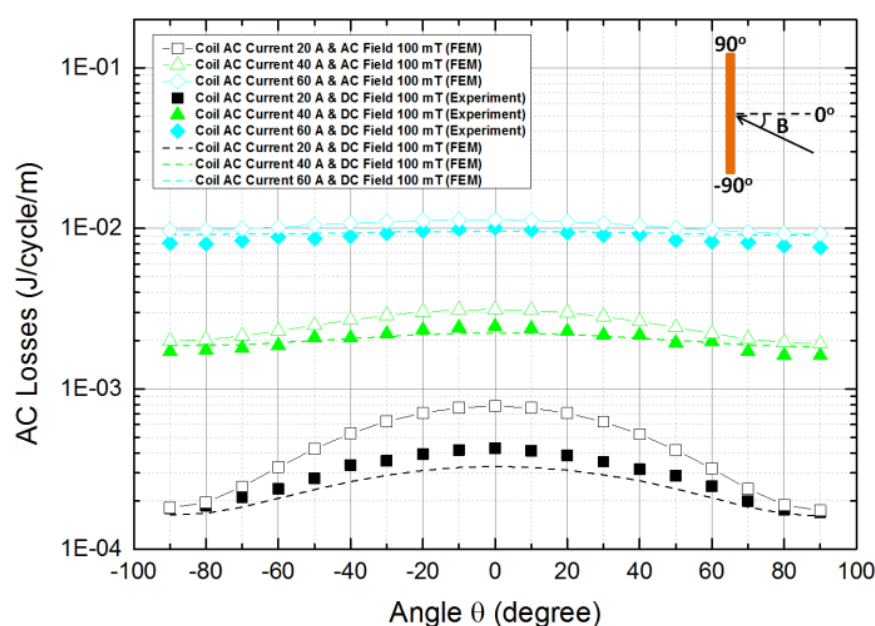

Figure 17. AC loss simulation of the coil with $\mathrm{AC}$ transport currents (20 A, $40 \mathrm{~A}$, and $60 \mathrm{~A}$ ) and the angular dependence of an external $\mathrm{AC}$ magnetic field of $100 \mathrm{mT}$, and its comparison to the $\mathrm{AC}$ loss experiment and simulation of the coil with AC transport currents $(20 \mathrm{~A}, 40 \mathrm{~A}$, and $60 \mathrm{~A})$ but an external DC magnetic field of $100 \mathrm{mT}$.

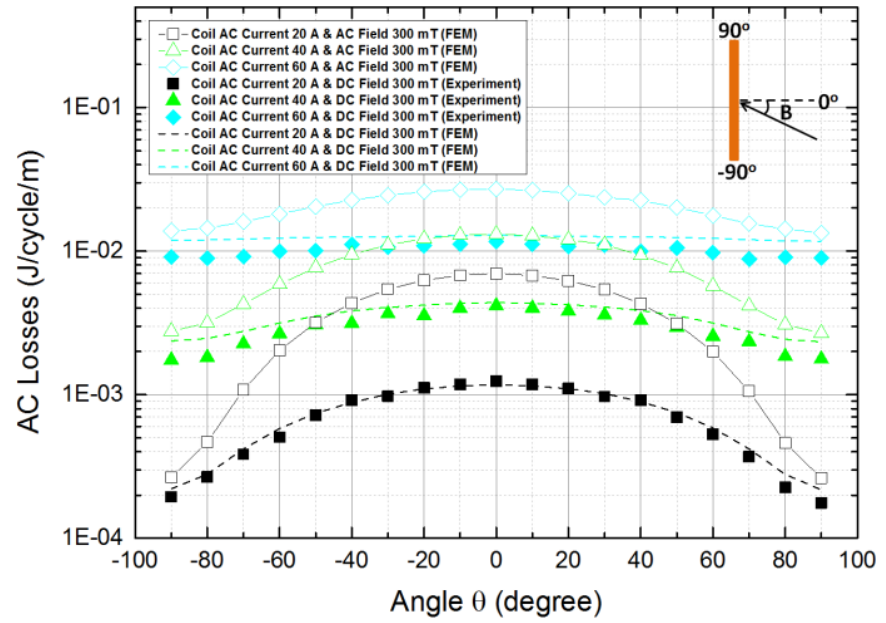

Figure 18. AC loss simulation of the coil with AC transport currents (20 A, $40 \mathrm{~A}$, and $60 \mathrm{~A}$ ) and the angular dependence of an external $\mathrm{AC}$ magnetic field of $300 \mathrm{mT}$, and its comparison to the $\mathrm{AC}$ loss experiment and simulation of the coil with AC transport currents (20 A, $40 \mathrm{~A}$, and $60 \mathrm{~A})$ but an external DC magnetic field of $300 \mathrm{mT}$.

current was more apparent than that with a higher DC transport current. For example, from the simulation with a $500 \mathrm{mT}$ AC field, the loss ratio of $\mathrm{Q}_{-}$odeg $/ \mathrm{Q}_{-} 90 \mathrm{deg}$ was 59.4 with an $\mathrm{AC}$ transport current of $20 \mathrm{~A}$, whilst the loss ratio of $\mathrm{Q}_{\_ \text {odeg }} / \mathrm{Q}_{-90 \mathrm{deg}}$ was 2.3 with an $\mathrm{AC}$ transport current of $60 \mathrm{~A}$. By comparing the two cases of external AC fields, $100 \mathrm{mT}$ and $500 \mathrm{mT}$, it can be noted that the angular dependence was stronger with a greater external AC field. By calculation with a 20 A AC transport current, the loss ratio of $\mathrm{Q}_{-} 0$ deg $/ \mathrm{Q}_{-90 \mathrm{deg}}$ was 4.5 with an AC magnetic field of $100 \mathrm{mT}$, and the loss ratio of $\mathrm{Q}_{\text {_odeg }} / \mathrm{Q}_{\text {_90deg }}$ was 59.4 with an $\mathrm{AC}$ magnetic field of $500 \mathrm{mT}$. To summarise, these two phenomena above occurred in all the three scenarios: Scenario $1 \mathrm{AC} I_{t} \&$ DC $B_{e x t}$, Scenario 2 DC $I_{t}$ \& AC $B_{e x t}$, and Scenario 3 AC $I_{t} \&$ AC $B_{e x t}$. However, for Scenarios 1 and 3, the AC loss with a higher transport current had much less angular dependence than the AC loss with a lower transport current, which is different from Scenario 2 where the angular dependence is still obvious with a higher transport current.

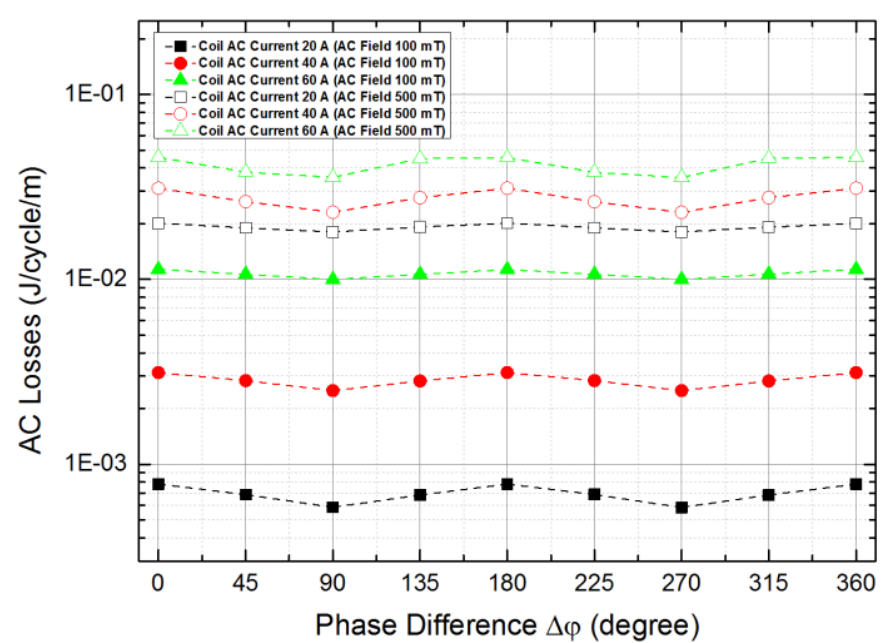

Figure 19. Phase difference $\Delta \varphi$ between the AC transport currents and the external AC magnetic field (from 0 to 360 degree): AC loss simulation of the coil with AC transport currents $(20 \mathrm{~A}, 40 \mathrm{~A}$, and $60 \mathrm{~A})$ and two sets of external AC magnetic fields of $100 \mathrm{mT}$ and $500 \mathrm{mT}$ perpendicular to the HTS tape surface of the coil.

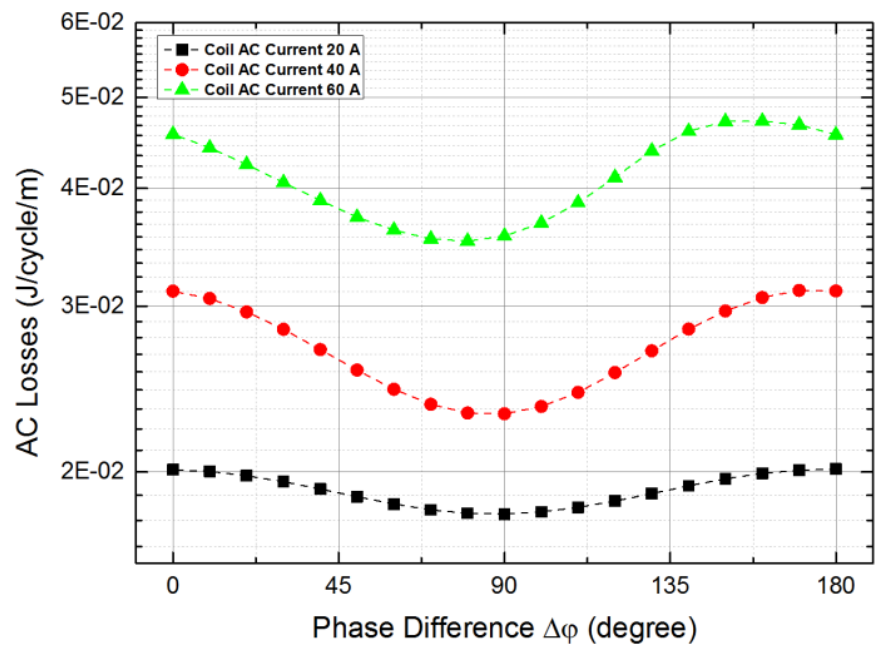

Figure 20. Phase difference $\Delta \varphi$ between the $\mathrm{AC}$ transport currents and external the AC magnetic field (from 0 to 180 degree): AC loss simulation of the coil with $\mathrm{AC}$ transport currents $(20 \mathrm{~A}, 40 \mathrm{~A}$, and $60 \mathrm{~A})$ and external $\mathrm{AC}$ magnetic field of $500 \mathrm{mT}$ perpendicular to the HTS tape surface of the coil.

It is helpful to compare Scenario 3 with Scenario 1 as both of them used the AC transport current but under different external fields (AC and DC, respectively). Figure 17 depicts the AC loss simulation of the coil with AC transport currents $(20 \mathrm{~A}, 40 \mathrm{~A}$, and $60 \mathrm{~A})$ and angular dependence of an external $\mathrm{AC}$ magnetic field of $100 \mathrm{mT}$, and its comparison to the $\mathrm{AC}$ loss experiment and simulation of the coil with $\mathrm{AC}$ transport currents $(20 \mathrm{~A}, 40 \mathrm{~A}$, and $60 \mathrm{~A})$ but an external DC magnetic field of $100 \mathrm{mT}$. It can be noted for all the three AC transport current cases, that the AC loss difference between the external $\mathrm{AC}$ and DC magnetic fields at $100 \mathrm{mT}$ was quite small when the field orientation angle $\theta$ was -90 or 90 degrees. For an AC transport current of $20 \mathrm{~A}$, the difference became larger when angle $\theta$ was 0 degree; but the difference was not significant for higher AC transport currents, e.g. only $15 \%$ for a $60 \mathrm{~A}$ transport current.

By contrast, in Figure 18, we plotted the AC loss simulation of the coil with $\mathrm{AC}$ transport currents $(20 \mathrm{~A}, 40 \mathrm{~A}$, and $60 \mathrm{~A}$ ) and the angular dependence of an external $\mathrm{AC}$ magnetic field of $300 \mathrm{mT}$, and its comparison to the AC loss experiment and 
simulation of the coil with the same AC transport currents but an external DC magnetic field of $300 \mathrm{mT}$. Similar phenomenon can be seen that when the field orientation angle $\theta$ was -90 or 90 degree, the AC loss difference between the external AC and DC magnetic fields at $300 \mathrm{mT}$ was slight. However, the difference significantly increased when the angle $\theta$ reached 0 degree, for all the three transport current cases, e.g. 5.9 times of a $20 \mathrm{~A}$ transport current case and 2.1 times of a $60 \mathrm{~A}$ transport current case. Therefore, one can summarise that for the HTS coil with AC transport current and in the presence of external DC or AC magnetic field with a given magnitude, the case of external AC magnetic field has a greater impact on its overall $\mathrm{AC}$ loss and angular dependence, particularly with stronger magnetic intensity.

\subsection{Phase difference $\Delta \varphi$ analysis with AC transport current and external AC magnetic field}

The above analyses were based on the assumption that both the AC transport current and the external AC magnetic field were in phase. However, if there was a phase difference $\Delta \varphi$, namely, AC transport current is leading or lagging the external $\mathrm{AC}$ magnetic field, the $\mathrm{AC}$ loss situation would change. Figure 19 illustrates the AC loss simulation of the coil with $\mathrm{AC}$ transport currents $(20 \mathrm{~A}, 40 \mathrm{~A}$, and $60 \mathrm{~A})$ and two sets of external AC magnetic fields of $100 \mathrm{mT}$ and $500 \mathrm{mT}$ perpendicular to the HTS tape surface of the coil. The phase difference between the AC transport currents and the external AC magnetic field, $\Delta \varphi$, was increased from 0 to 360 degree. As shown in Figure 19, the phase shift between AC field and $\mathrm{AC}$ transport current has some influence on the AC losses of the coil, which depends on the amplitude of current and field.

From Figure 19, it can be seen that for all the cases, the cycle of AC loss changing with $\Delta \varphi$ was 180 degree, and intuitively all the maximums occurred at $\Delta \varphi$ equal to 0,180 , and 360 degree, while all the minimums intuitively occurred at $\Delta \varphi$ equal to 90 and 270 degree. To be more precise, Figure 20 depicts the AC loss simulation of the coil with $\mathrm{AC}$ transport currents $(20 \mathrm{~A}, 40 \mathrm{~A}$, and $60 \mathrm{~A})$ and only one stronger external AC magnetic field of $500 \mathrm{mT}$ perpendicular to the HTS tape surface of coil, but with the phase difference $\Delta \varphi$ from 0 to 180 degree. It can be observed that, for the case of the $20 \mathrm{~A}$ transport current, the maximum loss happened when $\Delta \varphi$ was 0 and 180 , and the minimum loss happened when $\Delta \varphi$ was 90 . However, this kind of "90 degree symmetry" of loss characteristics changed when the $\mathrm{AC}$ transport increased to $40 \mathrm{~A}$ and $60 \mathrm{~A}$. As presented in Figure 20, the peak loss of $0.03118(\mathrm{~J} / \mathrm{cycle} / \mathrm{m})$ of the $40 \mathrm{~A}$ transport current case occurred at approximately $\Delta \varphi$ was 170 degree, and the peak loss of $0.04717(\mathrm{~J} / \mathrm{cycle} / \mathrm{m})$ of the $60 \mathrm{~A}$ transport current case occurred at approximately $\Delta \varphi$ was 160 degree. This kind of peak-shift phenomenon was more obvious with relatively stronger external AC magnetic fields, which is consistent with the measurements presented in [14].

\section{Conclusion}

A comprehensive study on AC losses in the circular HTS double pancake coil was carried out. We have established the experiment to measure the $\mathrm{AC}$ loss from a $2 \times 18$ circular double pancake coil using the electrical method. In order to enhance the consistency with the real circular coil used in the experiment, a 2D axisymmetric $\mathrm{H}$-formulation model using FEM package COMSOL Multiphysics was built.

We have investigated three scenarios which can cause the AC loss in a HTS coil: Scenario 1 AC transport current \& DC magnetic field; Scenario 2 DC transport current \& AC magnetic field; Scenario 3 AC transport current \& AC magnetic field. Moreover, the different orientation angle $\theta$ that HTS coil under the magnetic field has been studied for each scenario. For Scenario 3, the impact of relative phase difference between the AC current and the AC field on the total AC loss of coil was analysed. In short, we completed a current/field/angle/phase dependent AC loss $(\boldsymbol{I}, \boldsymbol{B}, \theta, \Delta \varphi)$ study of circular HTS coil, and we obtained the following results.

For Scenario 1 AC transport current \& DC magnetic field, both measurement and simulation, as well as their magnetic field angular dependency on AC loss of the coil were performed. The difference in AC loss with each set of external DC field became smaller when the transport current increased to a high value, which is consistent with the results reported in the literature. For the same transport current, the angular dependence increased with an increasing external DC field. For the same external DC field, the angular dependence with a smaller AC transport current was more significant than the angular dependence with a higher AC transport current. These results could be due to the fact that the external DC field is less influential on the reduction of critical current with the higher AC transport current conducting in the coil. Overall, the simulation results showed good agreement with experimental results.

For Scenario 2 DC transport current \& AC magnetic field, the simulation for the AC loss in the coil related to $\boldsymbol{I} / \boldsymbol{B} / \theta$, was performed. Two phenomena were similar to Scenario 1: (i) for the same transport current, the angular dependence increased with the an increasing external field; (ii) for the same external field, the angular dependence with a smaller transport current was more significant than with a higher transport current. However, in the presence of the same magnetic field, if increasing the DC transport current to a high value, it can be observed that the angular dependence did not change to much, which is different from Scenario 1. The AC loss increment tendency of Scenario 2 was different from Scenario 1, which was due to the fact that the AC losses were essentially caused by two different sources: AC transport current and $\mathrm{AC}$ external magnetic field.

For Scenario $3 \mathrm{AC}$ transport current \& AC magnetic field, the simulation for the AC loss in the coil related to $I / B / \theta$, was carried out. Again, it was found that the two phenomena (i) and (ii) mentioned above occurred in all three scenarios. We have also compared its angular dependency with the experimental and simulation results from Scenario 1: for the HTS coil conducting an AC transport current and in the presence of the same magnitude of external DC or AC magnetic field, the case of external AC magnetic field presented a greater impact on the overall $\mathrm{AC}$ loss and angular dependence, especially with stronger AC field. The effect of 
phase difference $\Delta \varphi$ between $\mathrm{AC}$ transport currents and external AC magnetic field was investigated. The phase difference $\Delta \varphi$ had a greater impact on the total AC losses either with a larger $\mathrm{AC}$ transport current or with a stronger external AC field. For further phase difference $\Delta \varphi$ analysis, the "90-degree symmetry" characteristics of AC loss changed when a larger AC transport current or a stronger external AC field were applied. These findings are consistent with reports in the literature.

Both the experiment and simulation were carried out in Scenario 1, and the good consistency (average error 3.2\%) of experiment and simulation was presented in terms of AC loss magnitude, tendency, and angular dependence. The simulation results of Scenario 2 and Scenario 3 were consistent with previous works presented in the literature. A powerful coil model has the potential to efficiently compute the AC loss from various complex conditions. To summarise, we have demonstrated a systematic study on AC losses from HTS coated conductor coils, and the methods and results of this study could be beneficial for future design and analysis on HTS AC systems.

\section{Acknowledgments}

The experimental work was carried out with the help of the Electrical Engineering Division, Department of Engineering, University of Cambridge. Authors are particularly grateful to Mr John Grundy and other members of staff for their crucial assistance. Some of the authors are research students, and they would like to express gratitude to China Scholarship Council (CSC) for their scholarships and support for overseas study.

\section{References}

[1] D. Larbalestier, A. Gurevich, D. M. Feldmann, and A. Polyanskii, "High-Tc superconducting materials for electric power applications," Nature, vol. 414, no. 6861, pp. 368-377, 2001.

[2] T. Tosaka, K. Koyanagi, K. Ohsemochi, M. Takahashi, Y. Ishii, M. Ono, H. Ogata, K. Nakamoto, H. Takigami, and S. Nomura, "Excitation tests of prototype HTS coil with Bi2212 cables for development of high energy density SMES," IEEE Trans. Appl. Supercond., vol. 17, no. 2, pp. 2010-2013, 2007.

[3] W. Schmidt, H.-P. Kraemer, H.-W. Neumueller, U. Schoop, D. Verebelyi, and A. P. Malozemoff, "Investigation of YBCO coated conductors for fault current limiter applications," IEEE Trans. Appl. Supercond., vol. 17, no. 2, pp. 3471-3474, 2007.

[4] H. S. Ruiz, X. Zhang, and T. Coombs, "Resistive-type superconducting fault current limiters: concepts, materials, and numerical modeling," IEEE Trans. Appl. Supercond., vol. 25, no. 3, pp. 1-5, 2015.

[5] S. S. Kalsi, Applications of high temperature superconductors to electric power equipment: John Wiley \& Sons, 2011.

[6] C. Rey, Superconductors in the power grid: Materials and applications: Elsevier, 2015.

[7] K. S. Haran, S. Kalsi, T. Arndt, H. Karmaker, R. Badcock, B. Buckley, T. Haugan, M. Izumi, D. Loder, and J. W. Bray, "High power density superconducting rotating machines-development status and technology roadmap," Supercond. Sci. Technol., vol. 30, no. 12, pp. 123002, 2017.

[8] F. Grilli, E. Pardo, A. Stenvall, D. N. Nguyen, W. Yuan, and F. Gömöry, "Computation of losses in HTS under the action of varying magnetic fields and currents," IEEE Trans. Appl. Supercond., vol. 24, no. 1, pp. 78-110, 2014.

[9] I. Hlasnik, "Review on AC losses in superconductors," IEEE Trans. Magn., vol. 17, no. 5, pp. 2261-2269, 1981.

[10] B. J. Parkinson, R. Slade, M. J. Mallett, and V. Chamritski, "Development of a cryogen free 1.5 T YBCO HTS magnet for MRI,"
IEEE Trans. Appl. Supercond., vol. 23, no. 3, pp. 4400405-4400405, 2013.

[11] N. Amemiya, K. Miyamoto, S.-i. Murasawa, H. Mukai, and K. Ohmatsu, "Finite element analysis of AC loss in non-twisted Bi-2223 tape carrying AC transport current and/or exposed to DC or AC external magnetic field," Phys. C, Supercond., vol. 310, no. 1, pp. 30-35, 1998.

[12] M. Ciszek, O. Tsukamoto, N. Amemiya, M. Ueyama, and K. Hayashi, "Angular dependence of AC transport losses in multifilamentary Bi2223/Ag tape on external DC magnetic fields," IEEE Trans. Appl. Supercond., vol. 9, no. 2, pp. 817-820, 1999.

[13] T. Chiba, Q. Li, S. Ashworth, M. Suenaga, and P. Haldar, "Angular dependence of ac losses at power frequencies for a stack of Bi2223/Ag tapes," IEEE Trans. Appl. Supercond., vol. 9, no. 2, pp. 2143-2146, 1999.

[14] D. Nguyen, P. Sastry, G. Zhang, D. Knoll, and J. Schwartz, "AC loss measurement with a phase difference between current and applied magnetic field," IEEE Trans. Appl. Supercond., vol. 15, no. 2, pp. 28312834, 2005.

[15] L. Ying, J. Xu, J. Sheng, B. Lin, Z. Jin, Z. Hong, and Z. Li, "Numerical and experimental analysis of AC loss of YBCO coated conductor carrying DC and AC offset transport current," IEEE Trans. Appl. Supercond., vol. 23, no. 3, pp. 5900704-5900704, 2013.

[16] V. Lahtinen, E. Pardo, J. Šouc, M. Solovyov, and A. Stenvall, "Ripple field losses in direct current biased superconductors: Simulations and comparison with measurements," J. Appl. Phys., vol. 115, no. 11, pp. $113907,2014$.

[17] B. Shen, J. Li, J. Geng, L. Fu, X. Zhang, H. Zhang, C. Li, F. Grilli, and T. A. Coombs, "Investigation of AC losses in horizontally parallel HTS tapes," Supercond. Sci. Technol., vol. 30, no. 7, 2017.

[18] F. Grilli, R. Brambilla, and L. Martini, "Modeling high-temperature superconducting tapes by means of edge finite elements," IEEE Trans. Appl. Supercond., vol. 17, no. 2, pp. 3155-3158, 2007.

[19] Z. Hong, A. M. Campbell, and T. A. Coombs, "Numerical solution of critical state in superconductivity by finite element software," Supercond. Sci. Technol., vol. 19, no. 12, pp. 1246, 2006.

[20] R. Brambilla, F. Grilli, and L. Martini, "Development of an edgeelement model for AC loss computation of high-temperature superconductors," Supercond. Sci. Technol., vol. 20, no. 1, pp. 16, 2006.

[21] Z. Hong, Q. Jiang, R. Pei, A. Campbell, and T. Coombs, "A numerical method to estimate AC loss in superconducting coated conductors by finite element modelling," Supercond. Sci. Technol., vol. 20, no. 4, pp. 331, 2007.

[22] B. Shen, L. Fu, J. Geng, X. Zhang, H. Zhang, Q. Dong, C. Li, J. Li, and T. A. Coombs, "Design and simulation of superconducting Lorentz Force Electrical Impedance Tomography (LFEIT)," Phys. C, Supercond., vol. 524, pp. 5-12, 2016.

[23] F. Grilli, F. Sirois, V. M. Zermeno, and M. Vojenčiak, "Self-consistent modeling of the Ic of HTS devices: How accurate do models really need to be?," IEEE Trans. Appl. Supercond., vol. 24, no. 6, pp. 1-8, 2014.

[24] R. Duckworth, M. Gouge, J. Lue, C. Thieme, and D. Verebelyi, "Substrate and stabilization effects on the transport AC losses in YBCO coated conductors," IEEE Trans. Appl. Supercond., vol. 15, no. 2, pp. 1583-1586, 2005.

[25] B. Shen, J. Li, J. Geng, L. Fu, X. Zhang, C. Li, H. Zhang, Q. Dong, J. $\mathrm{Ma}$, and T. A. Coombs, "Investigation and comparison of AC losses on Stabilizer-free and Copper Stabilizer HTS tapes," Phys. C, Supercond., vol. 541, 2017.

[26] F. Grilli, V. M. Zermeno, E. Pardo, M. Vojenčiak, J. Brand, A. Kario, and W. Goldacker, "Self-field effects and AC losses in pancake coils assembled from coated conductor Roebel cables," IEEE Trans. Appl. Supercond., vol. 24, no. 3, 2014.

[27] W. Norris, "Calculation of hysteresis losses in hard superconductors carrying ac: isolated conductors and edges of thin sheets," Journal of Physics D: Applied Physics, vol. 3, no. 4, pp. 489, 1970.

[28] F. Grilli, and S. P. Ashworth, "Measuring transport AC losses in YBCOcoated conductor coils," Supercond. Sci. Technol., vol. 20, no. 8, pp. 794, 2007. 